\title{
Expanding Clinical Utilization of Checkpoint Inhibitors for Cancer Treatments Necessitates the Development of Predictive Models for Immunotherapy-Induced Pneumonitis
}

\author{
Ivaylo B Mihaylov*, Christopher Montoya and Benjamin Spieler \\ University of Miami Miler School of Medicine, Department of Radiation Oncology, USA
}

*Corresponding author: Ivaylo B Mihaylov, University of Miami Miler School of Medicine, Department of Radiation Oncology, 1475 NW 12th Ave, Suite 1500, Miami, FL 33136, USA

\begin{tabular}{|c|c|}
\hline ARTICLE INFO & ABSTRACT \\
\hline $\begin{array}{l}\text { Received: 蔧 May 28, } 2021 \\
\text { Published: 㟵 June 04, } 2021\end{array}$ & $\begin{array}{l}\text { Citation: Ivaylo B Mihaylov, Christopher Montoya, Benjamin Spieler. Expanding Clinical } \\
\text { Utilization of Checkpoint Inhibitors for Cancer Treatments Necessitates the Development } \\
\text { of Predictive Models for Immunotherapy-Induced Pneumonitis. Biomed J Sci \& Tech Res } \\
\text { 36(2)-2021. BJSTR. MS.ID.005827. }\end{array}$ \\
\hline
\end{tabular}

\section{Opinion}

Immunotherapy has revolutionized treatment and improved prognosis for patients with a variety of advanced-stage cancers. Use of immune checkpoint inhibitors (ICIs) continues to increase due to this dramatic impact [1]. ICIs that target programmed cell death protein-1 (PD-1), programmed death protein ligand-1 (PDL1), and cytotoxic T-lymphocyte-associated antigen 4 (CTLA-4) have provided lasting responses and improved long-term survival in select patients with advanced non-small cell lung cancer (NSCLC) [2-6]. Despite this success, overall response rates to ICIs remain less than $50 \%$ and those patients who do not respond can experience accelerated and lethal disease progression $[7,8]$. In addition, ICI-mediated immune dysfunction may result in serious immunotherapy-related adverse events (irAEs) [9]. While irAEs can involve any organ system, gastrointestinal toxicities, pruritis, rash, hyphophysitis, vitiligo, dysthyroidism, hepatotoxicity, and pneumonitis are the most commonly observed complications [10]. The actual incidence of irAEs varies between agents and is likely underestimated $[9,11]$. Checkpoint-inhibitor pneumonitis (CIP) is a potentially life-threatening irAE more commonly seen among patients treated for NSCLC than for other malignancies $[12,13]$. Pneumonitis is defined as focal or diffuse inflammation of the lung parenchyma [14] and can occur with chemotherapy $[15,16]$, targeted therapy $[17,18]$ and radiotherapy (RT), as well as with ICIs [19]. Pre-ICI experiences with pneumonitis caused by cancer therapeutics emphasized that clinical and radiologic signatures may facilitate early recognition, treatment selection, and improvement in outcomes. Little is known about the etiology and molecular mechanisms underlying CIP [20]. While life-threatening CIP is rare, in NSCLC patients CIP can mimic conditions such as tumor progression or infection [11]. In the setting of expanding use of ICIs, clinicians should be educated regarding prompt CIP recognition and appropriate management.

Besides serious clinical consequences, pneumonitis contributes to treatment noncompliance, often requiring dose holds, immunosuppressive therapy, and/or permanent ICI cessation [12]. Most cases of CIP are mild and managed successfully in the outpatient setting, but inpatient management, long-term respiratory complications, or treatment-related deaths occur in $20 \%$ of affected patients [21-23]. The time to onset of pneumonitis has a wide range ( 9 days to 19.2 months with a median of 2.7 months for combination ICI and 4.6 months for ICI monotherapy) [12,22], complicating recognition and classification of CIP especially for NSCLC patients. Timely identification or, even better, pre-treatment prediction, could help to mitigate or prevent serious pulmonary complications. The natural history of CIP differs from other irAES; while thyroiditis or hepatitis are illnesses that tend 
to be self-limiting, most cases of CIP require immunosuppression with high dose steroids [24]. In a large retrospective study that reported overall incidence of CIP at $\sim 5 \%$, the majority of CIP cases improved with corticosteroids [12,25] but $9 \%$ of CIP patients died during immunosuppressive therapy due to infection or CIP itself, only $28 \%$ of patients whose CIP resolved were rechallenged with ICI, and $25 \%$ of the rechallenged cohort developed recurrent CIP and had to permanently discontinue immunotherapy.

In clinical trials for NSCLC, the incidences of all-grade CIP are typically reported at 5\% for ICI monotherapy and between 7-10\% for combination therapy $[13,26,27]$. However, the incidence of CIP in NSCLC outside of clinical trials remains uncertain, with CIP presentation mimicking infectious pneumonia or tumor progression and radiologic patterns of pneumonitis varying greatly from case to case [12]. For patients with NSCLC, CIP signs and symptoms can be misclassified in the setting of underlying lung disease and actual incidence may be higher than reported $[11,28]$. The ability to identify patients at risk for CIP prior to immunotherapy could prevent significant morbidity; discovery of signatures or methods for early CIP prediction represents an unmet clinical need.

Recent advances in analysis of medical imaging have made CIP risk prediction attainable. In the emerging technology known as quantitative imaging or radiomics, medical (PET, CT, MRI) images are analyzed at voxel level to detect textural features associated with disease or health [29]. Radiomics evaluate both semantic (volume, shape, etc.) and agnostic (texture) anatomical organ features. The former can be recognized by the naked eye while the latter require computer algorithms for detection at voxel level. Radiomics allow for comprehensive characterization of the tissue of interest and its corresponding microenvironment. Quantitative imaging has been used in recent years to predict tumor response at various anatomical sites [29-33]. Less attention has been devoted however to radiomics in the context of normal tissue complication predictions $[34,35]$.

The first publication [36] that used a radiomics model to predict CIP was a proof of principle study that retrospectively evaluated a small cohort of patients (30) treated with immunotherapy. Quantitative analysis of pre-immunotherapy baseline chest CT was able to identify the only two patients who went on to develop CIP. A study from a separate group [28] reviewed a larger cohort of NSCLC patients with higher incidence of clinically diagnosed CIP (6\%) after PD-1 checkpoint-inhibitor therapy. Retrospective chart review of CIP candidates demonstrated an incidence rate closer to $20 \%$, three times higher than commonly reported. These results were observed in a patient population receiving monotherapy, suggesting that actual CIP incidence could be even higher among patients treated with combination immunotherapy [12].

With increasing use of ICIs to treat advanced cancer, comprehensive CIP predictive models that incorporate uncorrelated but complementary biomarkers are poised for clinical application. Quantitative imaging is a promising analytic technique, but it need not stand alone; laboratory-derived parameters such as blood counts, blood inflammatory biomarkers, and inflammatory biomarkers in bronchoalveolar lavage fluid [37] as well as common clinical variables such as smoking history, comorbid conditions, and performance status promise to advance and refine CIP predictive modeling.

\section{References}

1. Kruger S, Ilmer M, Kobold S, Bruno L Cadilha, Stefan Endres, et al. (2019) Advances in cancer immunotherapy 2019 - latest trends. J Exp Clin Cancer Res 38(1): 268.

2. Borghaei H, Paz-Ares L, Horn L, David R Spigel, Martin Steins, et al. (2015) Nivolumab versus Docetaxel in Advanced Nonsquamous NonSmall-Cell Lung Cancer. The New England journal of medicine 373(17): 1627-1639.

3. Brahmer J, Reckamp KL, Baas P, Lucio Crinò, Wilfried E E Eberhardt, et al. (2015) Nivolumab versus Docetaxel in Advanced Squamous-Cell NonSmall-Cell Lung Cancer. The New England journal of medicine 373(2): 123-135.

4. Frederickson AM, Arndorfer S, Zhang I, Maria Lorenzi, Ralph Insinga, et al. (2019) Pembrolizumab plus chemotherapy for first-line treatment of metastatic nonsquamous non-small-cell lung cancer: a network metaanalysis. Immunotherapy 11(5): 407-428.

5. Gandhi L, Rodriguez-Abreu D, Gadgeel S, Emilio Esteban, Enriqueta Felip, et al. (2018) Pembrolizumab plus Chemotherapy in Metastatic Non-Small-Cell Lung Cancer. The New England journal of medicine 378(22): 2078-2092.

6. Rittmeyer A, Barlesi F, Waterkamp D, Keunchil Park, Fortunato Ciardiello, et al. (2017) Atezolizumab versus docetaxel in patients with previously treated non-small-cell lung cancer (OAK): a phase 3, open-label, multicentre randomised controlled trial. Lancet 389(10066): 255-265.

7. Champiat S, Dercle L, Ammari S, Christophe Massard, Antoine Hollebecque, et al. (2017) Hyperprogressive Disease Is a New Pattern of Progression in Cancer Patients Treated by Anti-PD-1/PD-L1. Clinical cancer research: an official journal of the American Association for Cancer Research 23(8): 1920-1928.

8. Chen DS, Mellman I (2017) Elements of cancer immunity and the cancerimmune set point. Nature. 541(7637): 321-330.

9. Thompson JA, Schneider BJ, Brahmer J, Stephanie Andrews, Philippe Armand, et al. (2020) NCCN Guidelines Insights: Management of Immunotherapy-Related Toxicities, Version 1.2020. Journal of the National Comprehensive Cancer Network: JNCCN 18(3): 230-241.

10. Kumar V, Chaudhary N, Garg M, Floudas CS, Soni P, et al. (2017) Current Diagnosis and Management of Immune Related Adverse Events (irAEs) Induced by Immune Checkpoint Inhibitor Therapy. Front Pharmacol 8:49.

11. Puzanov I, Diab A, Abdallah K, Clifton OB, C Brogdon, et al. (2017) Managing toxicities associated with immune checkpoint inhibitors: consensus recommendations from the Society for Immunotherapy of Cancer (SITC) Toxicity Management Working Group. J Immunother Cancer 5(1): 95.

12. Naidoo J, Wang X, Woo KM, Tunc I, Darragh H, et al. (2017) Pneumonitis in Patients Treated With Anti-Programmed Death-1/Programmed Death Ligand 1 Therapy. J Clin Oncol 35(7): 709-717.

13. Nishino M, Giobbie-Hurder A, Hatabu H, Ramaiya NH, Hodi FS (2016) Incidence of Programmed Cell Death 1 Inhibitor-Related Pneumonitis in Patients With Advanced Cancer: A Systematic Review and Meta-analysis. JAMA Oncol 2(12): 1607-1616. 
14. Disayabutr S, Calfee CS, Collard HR, Wolters PJ (2015) Interstitial lung diseases in the hospitalized patient. BMC Med 13: 245.

15. Genestreti G, Di Battista M, Trisolini R, Fabio D, Mirca V, et al. (2015) A commentary on interstitial pneumonitis induced by docetaxel: clinical cases and systematic review of the literature. Tumori 101(3): e92-95.

16. Poole BB, Hamilton LA, Brockman MM, Byrd DC (2014) Interstitial pneumonitis from treatment with gemcitabine. Hosp Pharm 49(9): 847850.

17. Liu V, White DA, Zakowski MF, G Michelle, Miller V, et al. (2007) Pulmonary toxicity associated with erlotinib. Chest 132(3): 1042-1044.

18. White DA, Camus P, Endo M, Bernard E, E Calvo, et al. (2010) Noninfectious pneumonitis after everolimus therapy for advanced renal cell carcinoma. Am J Respir Crit Care Med 182(3): 396-403.

19. Bradley J, Movsas B (2006) Radiation pneumonitis and esophagitis in thoracic irradiation. Cancer treatment and research 128: 43-64.

20. Kim ST, Sheshadri A, Shannon V, Dimitrios PK, Hagop K, et al. (2020) Distinct Immunophenotypes of T Cells in Bronchoalveolar Lavage Fluid From Leukemia Patients With Immune Checkpoint Inhibitors-Related Pulmonary Complications. Front Immunol 11: 590494.

21. Gettinger SN, Horn L, Gandhi L (2015) Overall Survival and Long-Term Safety of Nivolumab (Anti-Programmed Death 1 Antibody, BMS-936558, ONO-4538) in Patients with Previously Treated Advanced Non-SmallCell Lung Cancer. J Clin Oncol 33(18): 2004-2012.

22. Postow MA, Chesney J, Pavlick AC, Caroline Robert, Kenneth Grossmann, et al. (2015) Nivolumab and ipilimumab versus ipilimumab in untreated melanoma. The New England journal of medicine. 372(21): 2006-2017.

23. Topalian SL, Hodi FS, Brahmer JR, Scott N Gettinger, David C Smith, et al. (2021) Safety, activity, and immune correlates of anti-PD-1 antibody in cancer. The New England journal of medicine 366(26): 2443-2454.

24. Suresh K, Naidoo J, Lin CT, Danoff S (2018) Immune Checkpoint Immunotherapy for Non-Small Cell Lung Cancer: Benefits and Pulmonary Toxicities. Chest 154(6): 1416-1423.

25. Naidoo J, Page DB, Li BT, LC Connell, K Schindler, et al. (2016) Toxicities of the anti-PD-1 and anti-PD-L1 immune checkpoint antibodies. Annals of oncology: official journal of the European Society for Medical Oncology/ ESMO 27(7): 1362

26. Chuzi S, Tavora F, Cruz M, Ricardo Costa, Young Kwang Chae, et al. (2017) Clinical features, diagnostic challenges, and management strategies in checkpoint inhibitor-related pneumonitis. Cancer Manag Res 9: 207213.

\section{ISSN: 2574-1241}

\section{DOI: 10.26717/BJSTR.2021.36.005827}

Ivaylo B Mihaylov. Biomed J Sci \& Tech Res

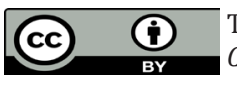

This work is licensed under Creative Commons Attribution 4.0 License

Submission Link: https://biomedres.us/submit-manuscript.php
27. Herbst RS, Baas P, Kim DW, Enriqueta Felip, José L Pérez-Gracia, et al. (2016) Pembrolizumab versus docetaxel for previously treated, PDL1-positive, advanced non-small-cell lung cancer (KEYNOTE-010): a randomised controlled trial. Lancet 387(10027): 1540-1550.

28. Spieler BO, Lopes G, Dal Pra A, Tejan Diwanji, Raphael Yechieli, et al. (2020) Is checkpoint inhibitor pneumonitis underreported in patients with advanced non-small cell lung cancer (NSCLC) on PD-1 inhibitor monotherapy? Journal of Clinical Oncology 38(15).

29. Gillies RJ, Kinahan PE, Hricak H (2016) Radiomics: Images Are More than Pictures, They Are Data. Radiology 278(2): 563-577.

30. Aerts HJ, Velazquez ER, Leijenaar RT, Chintan Parmar, Patrick Grossmann, et al. (2014) Decoding tumour phenotype by noninvasive imaging using a quantitative radiomics approach. Nature communications 5: 4006 .

31. Lambin P, Rios-Velazquez E, Leijenaar R, Sara Carvalho, Ruud GPM van Stiphout, et al. (2012) Radiomics: Extracting more information from medical images using advanced feature analysis. European journal of cancer 48(4): 441-446.

32. Lambin P, van Stiphout RG, Starmans MH, Emmanuel Rios-Velazquez, Georgi Nalbantov, et al. (2013) Predicting outcomes in radiation oncology-multifactorial decision support systems. Nature reviews Clinical oncology 10(1): 27-40.

33. Leijenaar RTH, Nalbantov G, Carvalho S, Wouter JC van Elmpt, Esther GC Troost, et al. (2015) The effect of SUV discretization in quantitative FDG-PET Radiomics: the need for standardized methodology in tumor texture analysis. Scientific reports 2015: 5.

34. Azzam G, Toomeh D, Kwon D, Mihaylov IB, Samuels S (2020) CT Dose-weighted Textures Indicate Radiation Induced Fibrosis (RIF) in Patients Treated for Head and Neck Cancers. Int J Radiat Oncol 108(3): E353-E353.

35. Meshman JJ, Toomeh D, Wang L, D Kwon, IB Mihaylov, C Takita, et al. (2020) Dose-Weighted CT Radiomics Associated with RadiationInduced Cardiac Toxicity in Women Treated for Localized Breast Cancer. Int J Radiat Oncol 108(3): E776-E777.

36. Colen RR, Fujii T, Bilen MA, Aikaterini Kotrotsou, Srishti Abrol, et al. (2017) Radiomics to predict immunotherapy-induced pneumonitis: proof of concept. Invest New Drugs doi: 10.1007/s10637-017-0524-2.

37.Zhong L, Altan M, Shannon VR, Sheshadri A (2020) Immune-Related Adverse Events: Pneumonitis. Adv Exp Med Biol 1244: 255-269.

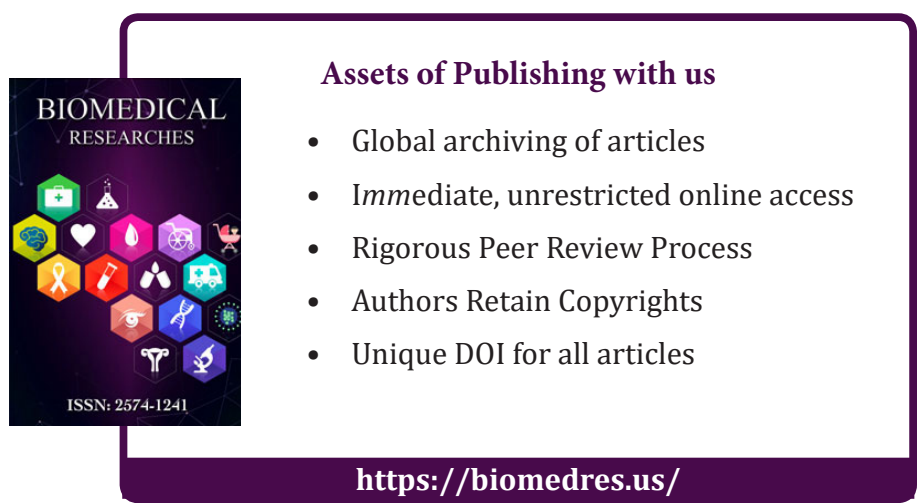

